\title{
Effect of rheological and structural properties of bacterial cellulose fibrils and whey protein biocomposites on electrosprayed food-grade particles
}

\author{
by Paximada, P., Kanavou, E. and Mandala, I.G.
}

Copyright, publisher and additional Information: This is the author accepted manuscript. The final published version (version of record) is available online via Elsevier.

This version is made available under the CC-BY-ND-NC licence: https://creativecommons.org/licenses/by-nc-nd/4.0/legalcode

Please refer to any applicable terms of use of the publisher

DOI: https://doi.org/10.1016/j.carbpol.2020.116319 
Manuscript Number: CARBPOL-D-20-00969R1

Title: Effect of rheological and structural properties of bacterial cellulose fibrils and whey protein biocomposites on electrosprayed foodgrade particles

Article Type: Research Paper

Keywords: Electrospraying; bacterial cellulose; Whey protein; particles; interfacial rheology

Corresponding Author: Dr. Ioanna Mandala, PhD

Corresponding Author's Institution: Agricultural University of Athens

First Author: Parskevi Paximada

Order of Authors: Parskevi Paximada; Eugenia Kanavou; Ioanna Mandala, PhD

Abstract: In this work, we investigated the role of bacterial cellulose nano-fibrils (BCNFs) as an alternative polymer to obtain food-grade particles with the electrospraying technique. Suspensions were prepared using BCNFs (1-16\% wt) and whey protein isolate (WPI) in various concentrations (10-30\% wt). Surface tension and electrical conductivity depended on the BC concentration and further increased by its increasing amount. A great increase in interfacial viscosity was also noticed according to the BCNFs concentration. A strong impact of BCNFs at the interface, influencing charge density and interactions of the two polymers was suggested. Different groups of the suspensions can be found that resulted in spherical nano- or submicron- particles by electrospraying. Uniform, nano-particles can be successfully produced taking into account the interfacial viscosity of the initial suspensions. Interfacial, compared to bulk viscosity, is a valuable tool to find out the appropriate suspension rheological properties in order to produce fine particles.

Research Data Related to this Submission

There are no linked research data sets for this submission. The following reason is given:

Data will be made available on request 
Highlights

- Spherical nano- or submicron- particles obtained through electrospraying

- Surface tension and viscosity capable to predict the structure of BC-WPI particles

- Interfacial viscosity of the suspensions related to the particle size and uniformity 
1 Effect of rheological and structural properties of bacterial cellulose fibrils and whey

2 protein biocomposites on electrosprayed food-grade particles

3 Paraskevi Paximada ${ }^{\mathrm{a}}$, Eugenia Kanavoua ${ }^{\mathrm{a}}$, Ioanna. G. Mandala ${ }^{\mathrm{a} 1}$

4

$5 \quad{ }^{a}$ Food Science \& Nutrition, Agricultural University of Athens, lera Odos 75, 11855, Athens,

6 Greece, imandala@aua.gr

7

${ }^{1}$ Corresponding author. Tel.: +302105294692

E-mail address: imandala@aua.gr (Ioanna Mandala) 


\section{Abstract}

2 In this work, we investigated the role of bacterial cellulose nano-fibrils (BCNFs) as an

3 alternative polymer to obtain food-grade particles with the electrospraying technique.

4 Suspensions were prepared using BCNFs (1-16\% wt) and whey protein isolate (WPI) in

5 various concentrations (10-30\% wt). Surface tension and electrical conductivity depended on

6 the $\mathrm{BC}$ concentration and further increased by its increasing amount. A great increase in

7 interfacial viscosity was also noticed according to the BCNFs concentration. A strong impact

8 of BCNFs at the interface, influencing charge density and interactions of the two polymers

9 was suggested. Different groups of the suspensions can be found that resulted in spherical

10 nano- or submicron- particles by electrospraying. Uniform, nano-particles can be successfully

11 produced taking into account the interfacial viscosity of the initial suspensions. Interfacial,

12 compared to bulk viscosity, is a valuable tool to find out the appropriate suspension

13 rheological properties in order to produce fine particles. 


\section{Introduction}

Cellulose is a natural polysaccharide as it is the primary component of plant cell walls.

Because of its fibrous structure and physical strength, it has high applicability in material industry e.g. paper and textile applications. In addition, bacterial cellulose (BC) is a natural polysaccharide, produced by bacteria. BC has remarkable properties compared to plantbased cellulose. The fibres of $\mathrm{BC}$ have higher aspect ratio, they are thinner, occupy larger area than plant-based cellulose fibres, and can absorb a great water amount creating a stiff 3D network. (Nakagaito, Iwamoto \& Yano, 2005; Nishi et al., 1990).

Commonly used hydrocolloids as thickeners are xanthan gum, locust bean gum, and hydroxymethylcellulose. Our previous studies concluded that BC shows a greater shear thinning behaviour than commercial thickeners (Paximada, Koutinas, Scholten \& Mandala, 2016). BC is more efficient to increase the zero-shear viscosity and the rheological profiles of $\mathrm{BC}$-stabilized emulsions, meaning that less amount of $\mathrm{BC}$ is required to obtain the same thickening-effect in a solution. Moreover, BC presents an exceptional bioaffinity resulting in several applications though it is still produced in lab scale (Choi \& Sin, 2020). Taking all the above into account, we can summarize that $B C$ is a cheaper alternative thickener.

Lately, protein and BCNF interactions have gained more attention in biocomposite production. For example, BCNFs and hydrolysed soy protein result in improved rheological properties, and cellulose fibrils enhance the solid-like behaviour of the dispersions (Gouzy,Tsekou, Remilin \& Velikov, 2019). Moreover, whey or soy protein isolates and BCNFs interact at the interface of an emulsion, stabilising the droplets. The investigated interfacial and bulk rheological properties give greater stability to the emulsion (Li et al., 2019; Paximada, Dubey \& Howarth, 2018). The complexes at the oil-water interface are formed due to the change of cellulose wettability. This wettability is caused by change of the adsorption capacity of the protein and polysaccharides.

Biopolymer particles may also be used to protect and deliver bioactive compounds in food systems. Therefore, there has been considerable interest in the formation of biopolymer nano-particles from proteins and/or polysaccharides. The most common techniques are spray-drying (Zhang et al., 2019), emulsification or coacervation (Koupantsis et., 2016). However, these techniques have significant drawbacks, such as the heating required for the 
process and the residual traces of solvents. To mitigate these drawbacks, electrohydrodynamic (EHD) processing and specifically electrospraying constitutes an innovative technology that could replace the commonly used techniques. In the electrospraying process, a high voltage is applied on a polymer solution drying it to particles that are then used in many applications (Bhardwaj \& Kundu, 2010). What is more, during electrospraying the particles are charged, hence they repulse each other leading to the elimination of particle agglomeration.

Electrospraying has distinctive advantages over conventional mechanical spraying systems such as the smaller droplet size with narrower distribution, higher stability of the bioactives. Parameters that influence the process are: polymer concentration, molecular weight and its distribution, solvent quality and volatility, physical properties (surface tension, conductivity, viscosity, viscoelasticity), and the equipment processing conditions (flow rate, distance between the electrodes and applied potential difference). These parameters have been analysed in many studies (Casper, Stephens, Tassi, Chase \& Rabolt, 2004, Regev, Vandebril, Zussman \& Clasen, 2010, Theron, Zussman \& Yarin, 2004).

Recently, proteins and polysaccharides have attracted significant attention in the search of suitable encapsulants to be electrosprayed. However, pure proteins are difficult or even impossible to be electrosprayed due to their three-dimensional network (López-Rubio and Lagaron, 2012). These limitations can be overcome by blending them with polysaccharides, such as starch (López-Rubio et al., 2012) or pullulan (Aceituno-Medina et al., 2013), which significantly improve the natural polymer's sprayability. Additionally, new polymers are particularly interesting molecules for electrospraying. Hence, this study focuses on the properties of bacterial cellulose and whey protein isolate mixtures in terms of their ability to be electrosprayed and to make particles for food applications. The properties of the produced particles are also within the scope of this study.

\section{Materials and methods}

\subsection{Materials}


1 Whey protein isolate (WPI), Lacprodan DI-9224, was kindly provided by Arla (Arla Foods 2 Ingredients, Amba-Denmark). Tween 20 was purchased from Fisher Scientific (St. Louis, 3 USA). All the other chemicals used were of analytical grade.

4 Bacterial cellulose (BC) was produced as described in our previous work (Paximada,

5 Koutinas, Scholten \& Mandala, 2016b). Briefly, bacterial cultivations (Komagataeibacter

6 sucrofermentans DSM 15973) were carried out using a synthetic medium. Acid hydrolysis in a

7 cellulose/acid ratio of approximately $20 \mathrm{~g} / \mathrm{L}$ took place in BC by adding sulfuric acid $(60 \% \mathrm{w} / \mathrm{w}$,

84 days, $40^{\circ} \mathrm{C}$ ) until homogenous suspension was obtained. BCNF were produced as a white

9 precipitate after several centrifugation (12.500 rpm) and washing cycles. The mean length of untreated cellulose fibrils was up to $37.5 \pm 6.8 \mu \mathrm{m}$ and their width up то $120 \pm 8 \mathrm{~nm}$. After acid treatment their length was $900 \pm 100 \mathrm{~nm}$ and their width $33 \pm 15 \mathrm{~nm}$. It should be mentioned that the differences between nanocrystals (BCNCs) and BCNFs, according to their length, can be slight. Lengths of 100-1500 nm are referred to crystals (Choi \& Sin, 2020), but we assume that the length of about $1 \mu \mathrm{m}$, is long enough to consider that nanofibrils are produced. Preliminary experiments showed that the type of acid, $\mathrm{HCl}$ or $\mathrm{H}_{2} \mathrm{SO} 4$, strongly affected the morphology of the produced fibrils. By using $\mathrm{H}_{2} \mathrm{SO} 4$ the smallest fibril size was achieved.

\subsection{Preparation of the suspensions}

BC-WPI suspensions were prepared by dissolving a precalculated amount of WPI powder and Tween 20 in the respective amount of BC suspension through gentle stirring for 2 hours at room temperature. The $\mathrm{pH}$ of the suspensions was adjusted at 3.6. The resulting suspensions reached a final concentration of $0-16 \%$ wt BC, $10-30 \%$ wt WPI, and $2 \%$ wt Tween 20.

\subsection{Electrospraying process}

For this process, an electrospraying set-up (Fluidnatek LE-10, Biolnicia S.L., Valencia, Spain) was used. This set up included a high voltage power supply. The suspensions were introduced in $5 \mathrm{~mL}$ syringes which were driven by a pump towards a stainless-steel needle (inner diameter $0.7 \mathrm{~mm}$ ). The needle was horizontal with respect to a grounded collector. The experiment was carried out at ambient conditions. The electrospraying conditions were fixed at $20-80 \mu \mathrm{L} / \mathrm{h}$ flow rate, $10-20 \mathrm{kV}$ voltage and a tip-to-collector distance of $10 \mathrm{~cm}$. 


\subsection{Characterization of the electrosprayed suspensions}

The surface tension and conductivity of the WPI-BCNFS suspensions were measured using a tensiometer (KSV Sigma 701, KSV Instruments Ltd., Finland) and a conductivity meter (SensoDirect 110, Lovibond, Dortmund, Germany), respectively. 弓-potential measurements were carried with Dynamic Laser Light Scattering (Zetasizer Nano ZS, Malvern Instruments, Worcestershire, UK) at $25^{\circ} \mathrm{C}$ using the method described previously (Paximada, Koutinas, Scholten \& Mandala, 2016).

\subsection{Rheological properties of the electrosprayed suspensions}

Rheological measurements of the suspensions were performed on a stress-controlled rheometer (Discovery HR-3, TA Instruments, New Castle, DE, USA) equipped with a concentric cylinders geometry (30mm cup diameter, $28 \mathrm{~mm}$ bob diameter) as described previously (Paximada, et al., 2016).

Moreover, the same rheometer was used to carry out interfacial rheological measurements. The geometry used was the Du Nouy ring (internal cup diameter $12.7 \mathrm{~mm}$, external cup diameter 25.6, internal ring diameter $19 \mathrm{~mm}$, external ring diameter 25.4, volume $4 \mathrm{ml}$ ). The shear rate was set between 0.1 and $100 \mathrm{~s}^{-1}$. The system was positioned at the interface and the sample was deliberately kept in direct contact with the ambient atmosphere. The soaking time of the sample was $10 \mathrm{~min}$.

\subsection{Microstructure and size of the particles}

The microstructure of the electrosprayed particles was determined using the SEM (JEOL JSM 6360 ) at an accelerating voltage of $20 \mathrm{kV}$ and a working distance of 9-12 mm, equipped with an image acquisition system. Samples were sputtered with a gold-palladium mixture under vacuum before examining their structure. Several pictures were taken from random sample positions representing the overall structure of each sample.

The particle size distributions were analyzed by Dynamic Laser Light Scattering equipment (Zetasizer Nano ZS, Malvern Instruments, Worcestershire, UK) using water as dispersing medium. Triplicate measurements were recorded at ambient temperature and the results are expressed as the mean value. The polydispersity index is also evaluated using the following equation:

$$
\mathrm{PDI}=\frac{\mathrm{D}_{90}-\mathrm{D}_{10}}{\mathrm{D}_{50}} \quad \text { (Eq. 1). }
$$




\subsection{Statistical analysis}

Statistical analysis of the results was performed with Statgraphics Centurion XV

(Statgraphics, Rockville, MD, USA) and an ANOVA test was applied in order to compare the mean values of selected properties at a $95 \%$ confidence level. The same software was used to perform clustering analysis, principal component analysis (PCA) and Pearson's multiple variable analysis.

\section{Results and discussion}

\subsection{Physical properties of the BCNFs-WPI suspensions}

Existing work has shown that the physical properties of polymer suspensions such as viscosity, surface tension and conductivity play a key role in the successful formation of particles (Kriegel, McClements \& Weiss, 2009). From Table 1, it can be observed that a drastic increase in the surface tension occurs by increasing the BCNFS concentration of the suspension. Specifically, for small amounts of BCNFS (1\% wt) the surface tension bears values of $20 \mathrm{mN} / \mathrm{m}$, while for higher ones (16\% wt BCNFS) the values go up to $40 \mathrm{mN} / \mathrm{m}$, regardless of the WPI concentration.

Table 1. Physical properties (surface tension, conductivity and $\zeta$-potential) of suspensions containing $1-16 \%$ wt BCNFs and $10-30 \%$ wt WPI and the polydispersity index (PDI) of the produced particles

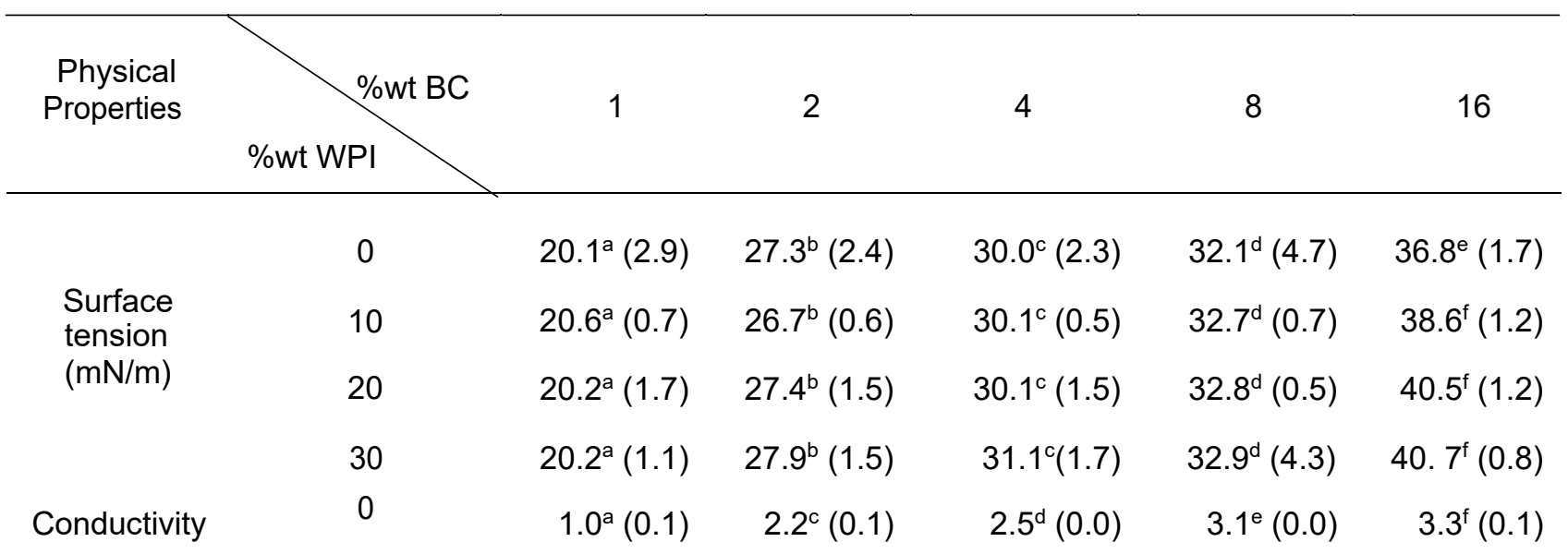




\begin{tabular}{ccrrrrr}
$(\mathrm{mS} / \mathrm{m})$ & 10 & $1.3^{\mathrm{b}}(0.0)$ & $2.4^{\mathrm{d}}(0.0)$ & $2.5^{\mathrm{d}}(0.0)$ & $3.1^{\mathrm{e}}(0.2)$ & $3.3^{\mathrm{f}}(0.1)$ \\
& 20 & $1.4^{\mathrm{b}}(0.1)$ & $2.5^{\mathrm{d}}(0.2)$ & $2.9^{\mathrm{e}}(0.1)$ & $3.1^{\mathrm{e}}(0.1)$ & $3.3^{\mathrm{f}}(0.0)$ \\
& 30 & $1.4^{\mathrm{b}}(0.0)$ & $2.6^{\mathrm{d}}(0.0)$ & $2.9^{\mathrm{e}}(0.2)$ & $3.1^{\mathrm{e}}(0.0)$ & $4.0^{\mathrm{g}}(0.2)$ \\
& 0 & $-20.6^{\mathrm{e}}(0.7)$ & $-21.5^{\mathrm{f}}(1.4)$ & $-21.6^{\mathrm{f}}(1.5)$ & $-25.9^{\mathrm{h}}(0.4)$ & $-28.3^{\mathrm{i}}(0.8)$ \\
$\begin{array}{c}\zeta \text {-potential } \\
(\mathrm{mV})\end{array}$ & 10 & $-18.3^{\mathrm{c}}(0.9)$ & $-19.7^{\mathrm{d}}(0.3)$ & $-20.5^{\mathrm{e}}(0.6)$ & $-21^{\mathrm{e}}(0.4)$ & $-23.1^{\mathrm{j}}(1.0)$ \\
& 20 & $-15.3^{\mathrm{b}}(1.2)$ & $-17.1^{\mathrm{c}}(0.9)$ & $-17.3^{\mathrm{c}}(1.8)$ & $-17.8^{\mathrm{c}}(2.1)$ & $-18.8^{\mathrm{c}}(0.8)$ \\
& 30 & $-11.9^{\mathrm{a}}(0.7)$ & $-12.6^{\mathrm{a}}(1.4)$ & $-14.1^{\mathrm{b}}(1.3)$ & $-14.6^{\mathrm{b}}(0.4)$ & $-15.9^{\mathrm{b}}(0.7)$ \\
& 0 & - & & - & - & - \\
PDI (-) & 10 & $0.8^{\mathrm{a}}(0.0)$ & $1.0^{\mathrm{b}}(0.0)$ & $0.9^{\mathrm{ab}}(0.0)$ & $0.9^{\mathrm{ab}}(0.0)$ & $1.0^{\mathrm{b}}(0.0)$ \\
& 20 & $0.9^{\mathrm{ab}}(0.0)$ & $0.9^{\mathrm{ab}}(0.0)$ & $1.1^{\mathrm{c}}(0.0)$ & $1.7^{\mathrm{d}}(0.1)$ & $2.1^{\mathrm{e}}(0.1)$ \\
& 30 & $1.0^{\mathrm{b}}(0.0)$ & $1.0^{\mathrm{c}}(0.0)$ & $1.6^{\mathrm{d}}(0.1)$ & $2.6^{\mathrm{f}}(0.2)$ & $3.1^{\mathrm{g}}(0.3)$ \\
\hline
\end{tabular}

1 In parenthesis standard deviation values.

2 Mean values followed by the same letters are not significantly different $(P>0.05)$.

3

$4 \quad$ Hydrophilicity is connected to lower contact angle and higher surface tension. It is also

5 mentioned that $\mathrm{BC}$ fibrils can create many inter-and intra-fibrillar hydrogen bonds reducing the

6 fibrils dimensions and increasing hydrophilicity (Jia et al., 2016). According to Ganzevles

7 (2007) the presence of an excess of anionic polysaccharide can hinder the formation of a

8 dense protein layer at the air/water interface, justifying the similar values observed by

9 increasing WPI concentration.

10 Surface tension values are of high importance as they play a major role in the final size of the

11 particles. Furthermore, differences in the size of the processed system could be mainly

12 attributed to changes in the surface tension of the suspensions. Specifically, a decrease in

13 surface tension from 40 to $20 \mathrm{mN} / \mathrm{m}$ at suspensions containing 16 to $1 \%$ wt BCNFs and

14 constant amount of WPI ( $20 \% \mathrm{wt})$, leads to a decrease of the particle size from 390 to roughly $15300 \mathrm{~nm}$ (Fig 1). 


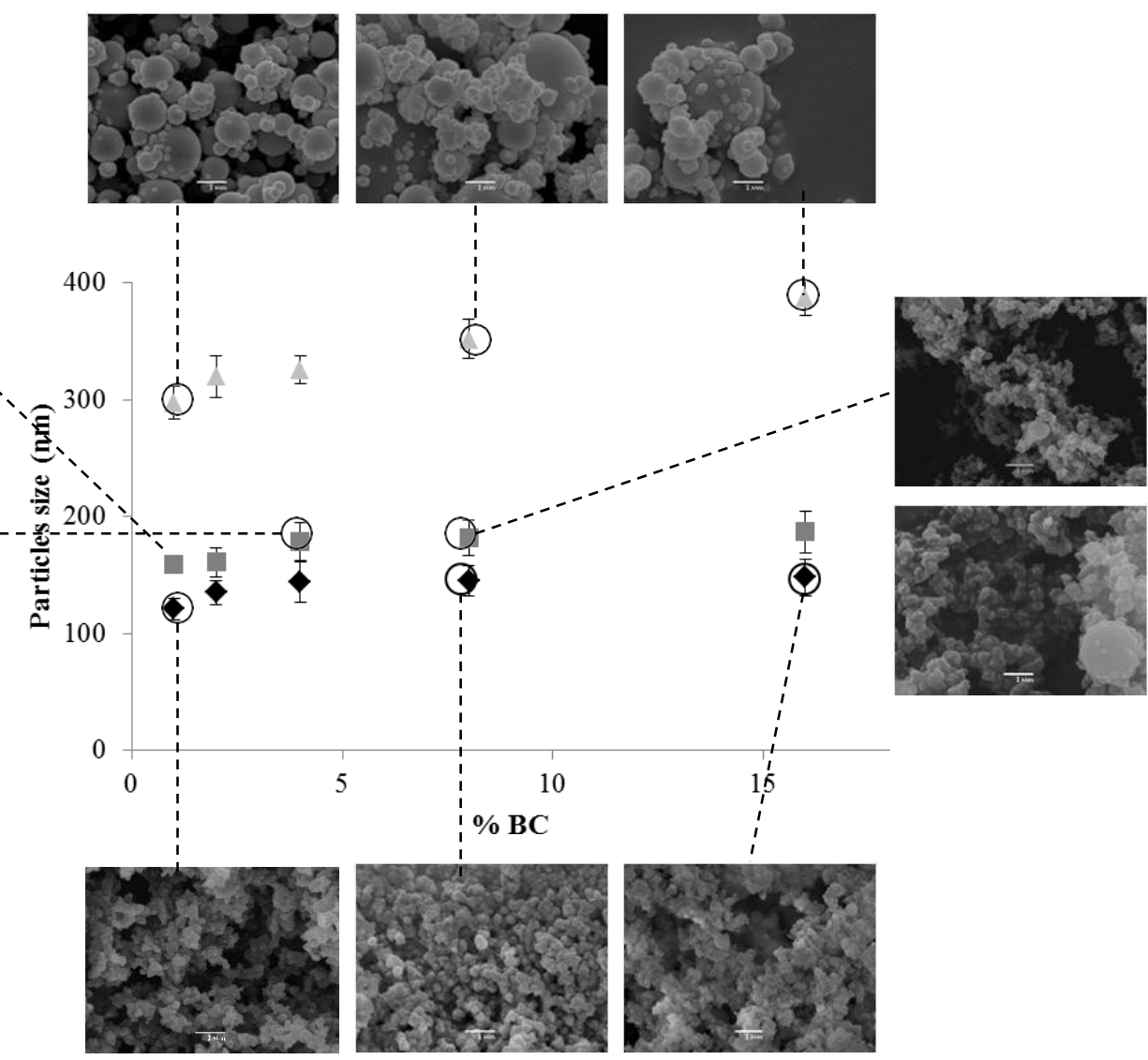

Fig. 1. Mean particle size $\left(d_{50}\right)$ and microstructure (scale $1 \mu \mathrm{m}$ ) of the different BCNFS-WPI particles obtained through electrospraying of 1-16\% wt BCNFs and 10\% (๑), 20\% (०), 30 (प ) wt \% WPI suspensions.

This observation could have been due to the lower surface tension gradient around the droplet that causes the formed Taylor's cone to be expelled from the needle. It is found that the diameter of the particles could only be reduced with the stable cone-jet mode, which also improves the size of the particles. Moreover, Pancholi, Arhas, Stride \& Ediringhe (2009) described a decrease of the mean particle size of chitosan particles, by decreasing the surface tension from 80 to $50 \mathrm{mN} / \mathrm{m}$. A more hydrophobic surfactant was used for that purpose.

According to our previous study (Kaltsa, Paximada, Mandala \& Scholten,2014) pure WPI solution reach an interfacial pressure of $26 \mathrm{mN} / \mathrm{m}$ after a certain time. However, the surface tension of BCNFs-WPI suspensions does not significantly vary with the WPI concentration (Table 1)..

This result is attributed to the fact that BCNFs with their containing proteins move faster and cover the interface and then only a small amount of WPI is sufficient in saturating the surface. 
1 When higher amounts of WPI are added to the system (20-30\% wt) the excess protein 2 remains in the suspension and does not affect the surface tension. This is consistent with 3 other studies that worked with proteins and found no effect of the amaranth proteins on the

4 surface tension (Aceituno-Medina, Mendoza, Lagaron \& Lopez-Rubio, 2013).

5 The conductivity values of the suspensions followed a steady increasing trend when the BCNFS concentration increased throughout the whole experiment (Table 1). Specifically, by increasing the BCNFS concentration from 1 to $16 \%$ wt, the electrical conductivity increased from 1.3 to $3.3 \mathrm{mS} / \mathrm{m}$, regardless of the WPI content. This phenomenon is attributed to the predominant effect that the addition of higher BCNFs concentrations has to the system, as it was noticed in surface tension values as well. In accordance to these results, Rošic, et al. (2012) found an increase in conductivity by increasing polysaccharide concentration. Conductivity is influenced by the polymer concentration because both natural polymers display polyelectrolyte properties in aqueous media, i.e., WPI is positively charged in citrate buffer and BCNFS is negatively charged in all the $\mathrm{pH}$ range.

15 The formation of a Taylor cone for electrospraying depends on the sufficiently high surface charge densities. In turn, these densities depend on the conductivity of the biopolymer suspension and the applied voltage (Yu, et al., 2011). Differences in the conductivity of BCNFs are relatively small and do not explain the observed large changes in particles diameter (Fig 1). On the other hand, the $30 \%$ wt WPI suspensions and high BCNFS amounts that exhibit high conductivity values, led to a complicated process. It is worth noting that if electrical conductivity is too high, the charge carried by the electrospraying jet is lower, resulting in potential destabilization of the jet and the complication of the process (Bock, 23 Dargaville \& Woodruff, 2012).

24 The $\zeta$-potential representing the charge density of the BCNFs-WPI suspensions is summarized in Table 1. It has been previously reported that $\mathrm{BC}$ bears a negative charge in all the $\mathrm{pH}$ range (Paximada, Koutinas, Scholten \& Mandala, 2016). Therefore, by increasing the BCNFs concentration from 1 to $16 \% \mathrm{wt}$, the overall charge of the suspensions decreased from -18 to $-23 \mathrm{mV}$ for suspensions containing $10 \% \mathrm{wt} \mathrm{WPI}$. The same trend is also obvious in suspensions containing 20 or $30 \%$ wt WPI. 
1 A progressive increase of WPI concentration from 10 to $30 \%$, at constant BCNFs amount,

2 leads in $\zeta$-potential increase in all cases. It is known that, at low $\mathrm{pH}$, since WPI is positively

3 charged, with $\zeta$-potential equal to $+35.9 \mathrm{mV}$ in emulsions systems at $\mathrm{Ph}=3.0$ according to

4 Kaltsa, Paximada, Mandala \& Scholten (2014). Then the increase in $\zeta$ - potential is attributed to

5 protein and BCNFs associations. However, positive values were not observed. This may

6 happen because $\mathrm{BC}$ small fibrils, due to their large volume, determine the overall charge

7 density. Moreover, the rate of $\zeta$-potential increase differs according to the initial BC amount. In

8 particular, at high BCNFs concentration, $8 \%$ or $16 \%$, the change of $\zeta$-potential is steeper. On

9 the contrary, at BCNFs concentration $1-4 \%$, this rate is similar and lower. When the protein

10 amount is constant but $B C$ increases, a slight decrease in the overall charge is observed.

11 Then the protein increase results in greater changes in $\zeta$ - potential than $B C$ does, in

12 agreement to further results when particles characteristics are categorized according to

13 protein amount (cluster analysis, 3.4).

14 To conclude, BC has a dominant effect on the surface tension and conductivity, whereas WPI

15 influenced $\zeta$-potential of the suspensions, leading to differences in the electrospraying

16 process and the produced particles.

17

18

3.2 Bulk viscosity of the BCNFs-WPI suspensions

19 The viscosity of the suspensions as a function of different BCNFs and WPI concentrations is 20 presented in Fig. 2 (A-C).

21 

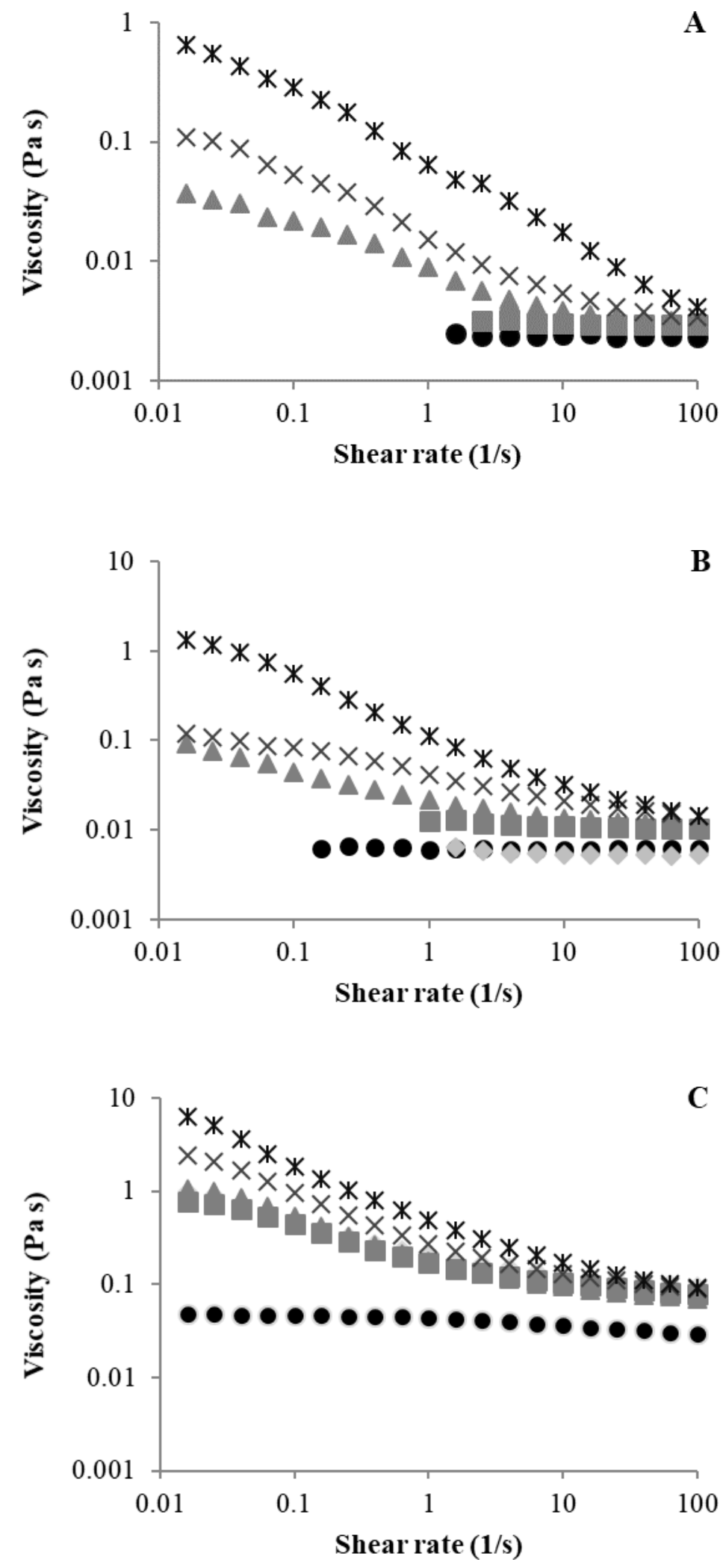

1 Fig. 2. Apparent viscosity curves of suspensions containing $0 \%$ wt $\left(\varsigma_{0}\right), 1 \% \mathrm{wt}(\odot), 2 \% \mathrm{wt}(\mathrm{O})$, 4\% wt ( $\mathrm{\square}$ ), 8\% wt (×), 16\% wt ( $\rightarrow$ ) BCNFs and 10\% (A), 20\% (B), 30\% (C) wt WPI. 
In the absence of BCNFs, all suspensions exhibit a Newtonian-like behavior as their viscosity is constant in all shear rates, regardless of the WPI concentration. This Newtonian-like behavior is typical for suspensions with a low WPI content (Paximada, Koutinas, Scholten \& Mandala, 2016). The viscosity of suspensions containing 10 or $20 \%$ wt and low amount of BCNFs (1-4\% wt) still exhibits Newtonian-like behavior, which is consistent with the idea that in suspensions contain low amounts of biopolymers, WPI is the polymer that has the major effect on the viscosity of the mixture. This has already been described from other studies in the properties of protein and polysaccharide mixtures (Kaltsa, Paximada, Mandala \& Scholten, 2014). For the rest of the suspensions, their viscosity increases significantly from roughly $7 \mathrm{mPas}$ (no BCNFS) to $0.7-7$ Pas, an increase of 2 to 3 orders of magnitude when BCNFS is from $1-16 \%$. The suspensions also exhibit shear thinning behavior, like most suspensions containing a high concentration of polymers. A synergistic interaction between the anionic BCNFs and the cationic WPI mixtures can be attributed either to the intermolecular binding that occurs between side chains of the polymers, or to the interactions between disordered segments of WPI molecules and BCNFs. This interaction enhances the viscosity of the suspensions (Renou, Petibon, Malhiac \& Grisel, 2013). The pseudoplasticity of the suspensions increases with an increasing proportion of BCNFs in the mixtures. The pronounced shear-thinning effect can be related to the structure of the polymer chains in the suspensions. Before the beginning of the shearing, each macromolecule has the shape of a three-dimensional coil as this is at the lowest energy state. During the shear process, the molecules are tent to orient parallel to the direction of shear, resulting in elongation, which lowers their flow resistance and results in a decrease in the bulk viscosity (Freeman, 1996). Viscosity is one of the crucial parameters in predicting the ability of one suspension to be electrospun or electrosprayed. High and low viscosities give rise to dripping of suspensions, which is the case in the suspensions containing $30 \%$ WPI (Fig.1). Studies that have examined different polymers on their ability to form particles, showed viscosities at the range of 50-250 mPas for chitosan (Pancholi, Ahras, Stride \& Edirisinghe, 2009), and 165 mPas for zein (Neo et al., 2013). At 16\% BCNFS concentration and at 30\% 
2 Numerous existing papers on electrosprayed or electrospun materials examined the viscosity

3 of the polymer suspensions (Aceituno-Medina, Mendoza, Lagaron \& Lopez-Rubio, 2013;

4 Sarhan and Azzazy, 2015). Interfacial viscosity may bear an effect on the sprayability of the

5 polymers (Pelipenko et al., 2012) suggesting that, given the area of a Taylor cone, rheological

6 characteristics play a significant role due to the larger diameter of the jet. Hence,

7 measurement of the rheological parameters of the bulk may not be sufficient when dealing

8 with electrospraying. In this study the interfacial viscosity of the suspensions as a function of

9 different BCNFS-WPI concentrations is evaluated and summarized in Fig. 3 (A-C).
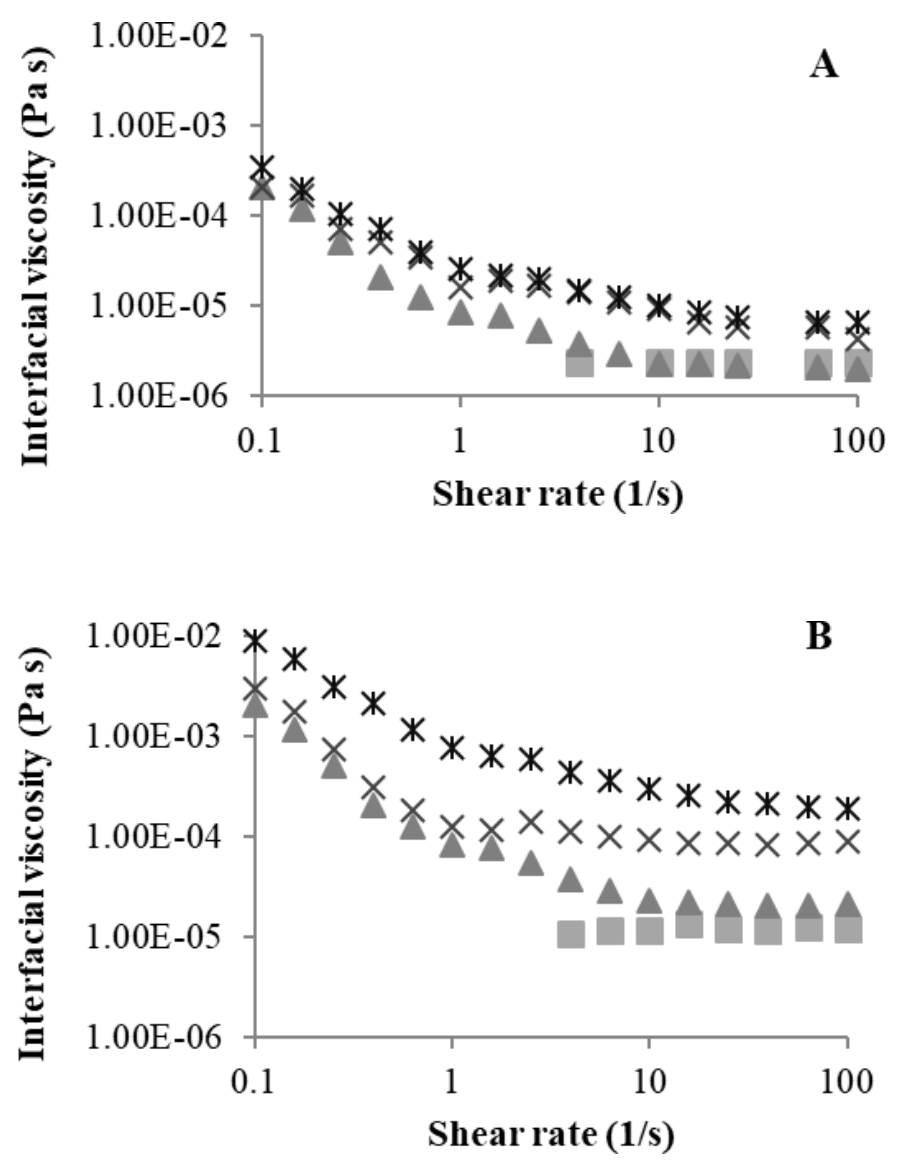


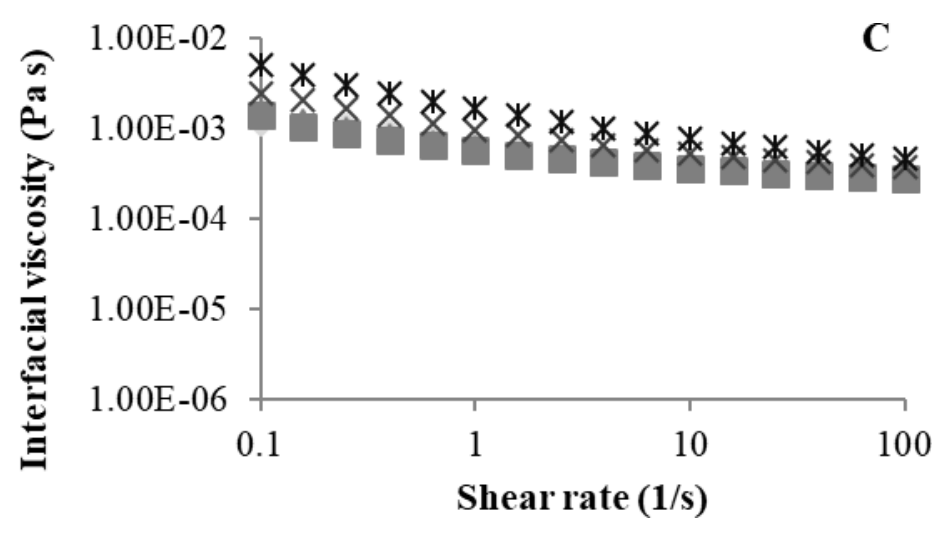

1 Fig. 3. Interfacial viscosity curves of suspensions containing $0 \% \mathrm{wt}(\infty)), 1 \% \mathrm{wt}(\oplus), 2 \% \mathrm{wt}(0)$, 4\% wt ( $\square$ ), $8 \%$ wt (x), 16\% wt $(\rightarrow)$ BCNFS and 10\% (A), 20\% (B), 30\% (C) wt WPI.

3

4 The results of the interfacial measurements presented in Fig. 4 show the same parameter

5 trends as was observed in the bulk (Fig.3 A-C). However, the values of the interfacial

6 viscosity are three to five orders of magnitude lower than those observed in the bulk, with the 7 latter being comparable to data obtained by Rošic et al. (2012).

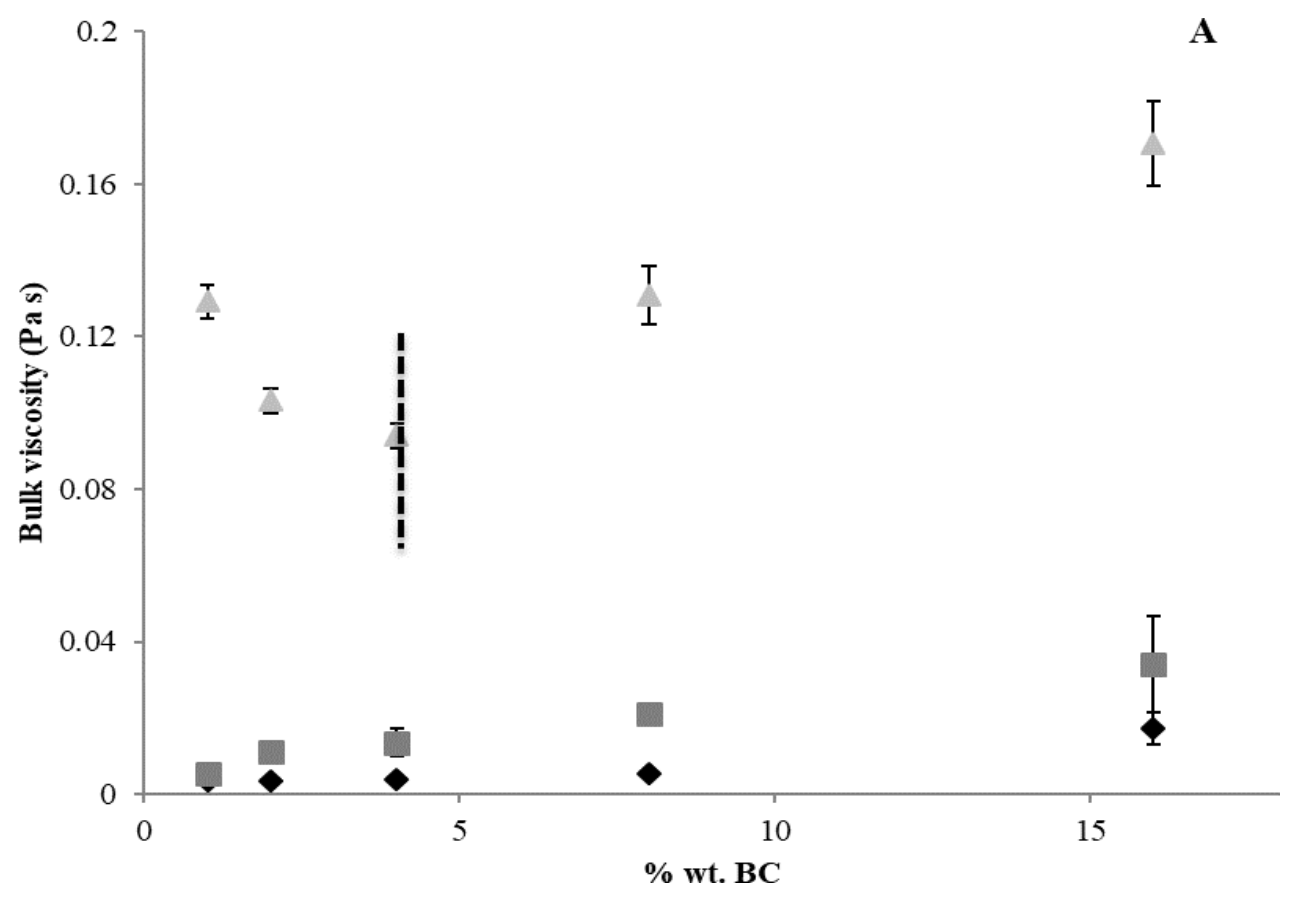




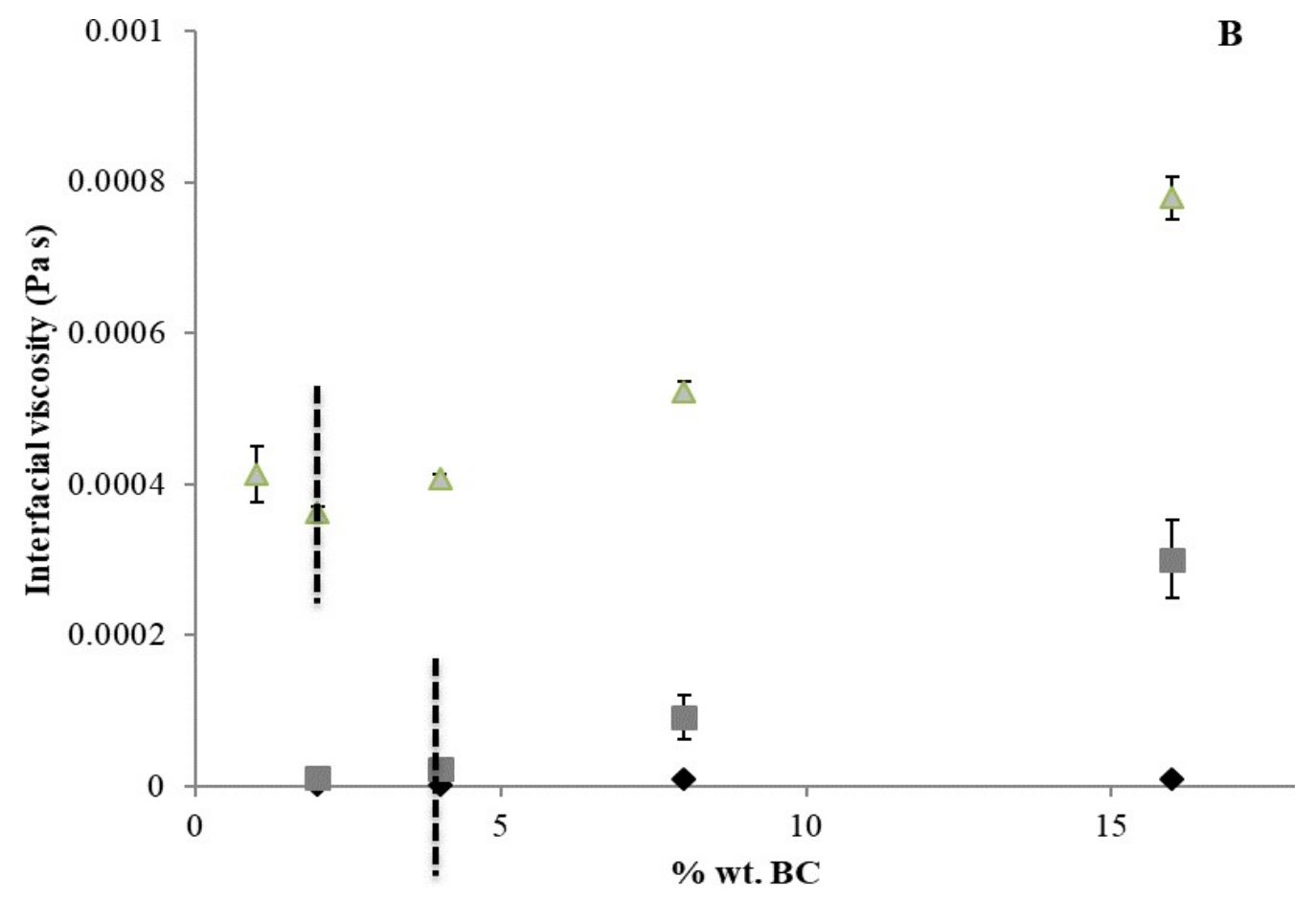

Fig. 4. Bulk (A) and interfacial viscosity curves (B) as a function of blended suspensions containing $1-16 \%$ wt BCNFs and 10\% wt (๑), $20 \%$ (०) or 30\% (口 ) wt WPI. Bars indicating standard deviations.

4 Specifically, the viscosity of all suspensions containing BCNFS increases significantly from roughly $0.001 \mathrm{mPas}(1 \% \mathrm{BCNFS})$ to $5 \mathrm{mPas}(16 \% \mathrm{BCNFS})$ regardless of the amount of WPI

6 that they contain. This increase in interfacial viscosity is attributed to the amount of BCNFs

7 that is added to the system. Hence, BC acts as a thickener in the suspensions and its 8 increase in both bulk and interfacial viscosity is in agreement with other studies that evaluated 9 the thickening effect of BC (Panagopoulou, et al., 2015; Paximada et al., 2016a; Paximada et al., 2016b). Fig 4 illustrates the bulk and interfacial viscosity values (Fig.4A, 4B) as a function

11 of the concentration of BCNFS in the suspensions. The values used for this graph were for both viscosities at shear rate of $10 \mathrm{~s}^{-1}$. From this figure, it can be seen that, while for the suspensions containing $10 \%$ wt WPI the bulk and interfacial viscosity do not have significant 14 differences, when the concentration of WPI is increased $(20-30 \% \mathrm{wt})$ high differences are found. Specifically, the interfacial viscosity for the $20 \%$ wt WPI suspensions can be divided in 162 regions: one from $1-4 \%$ wt BCNFs and the other from $4-16 \%$ wt BCNFs. As far as the 17 suspension containing $30 \%$ wt WPI is concerned, the bulk viscosity shows a 2-region profile, 
1 with the first region being from $1-4 \%$ wt and the second between $4-16 \%$ wt BCNFs. The

2 interfacial viscosity values of the first region were between $1-2 \%$ wt and between $2-16 \%$ wt of

3 BCNFs for the second.

4 A good correlation can be made between the viscosity values (Fig. 2A-C) and the polydispersity index (PDI) of the particle's diameter (Table 1). In the case of $10 \%$ wt WPI, only one region is depicted for both the bulk and interfacial viscosity values (Fig 5A, B), while the PDI of all the produced samples was below 1(Eq. 1). A PDI lower than 1, confirms the monomodal size distribution of the particles (McClements, 2005). Another issue to consider is the viscosity of the suspensions with $20 \% \mathrm{WPI}$. Only one region is observed for the bulk viscosity (Fig. 4(A, B)). However, for the interfacial viscosity, 2 regions are observed, and they are in agreement with the polydispersity of the suspensions which showed that from $1-4 \%$ wt BCNFS the distribution is monomodal. However, by increasing the BCNFS amount, the distribution becomes bimodal. Regarding the 2 regions observed for $30 \% \mathrm{wt} W P I$, the slope in the first region includes suspensions having $4 \%$ wt or less BCNFS in the suspension, while the second region includes BCNFS content of up to $16 \% \mathrm{wt}$. In contrast to the results of the bulk viscosity, interfacial viscosity (Fig. 4B) exhibits 2 regions, the first being for BCNFS concentrations up to $2 \% \mathrm{wt}$ and the second up to $16 \% \mathrm{wt}$. The interfacial viscosity values are in better agreement with the polydispersity of the produced particles. Suspensions falling in the first region gave particles of monomodal size distribution $(P D \mid<1)$, while those falling in the second region resulted in particles that exhibit a multimodal distribution as their PDI was greater than 1. Specifically, the absolute value of the correlation for bulk viscosity and PDI is lower (0.68) than the values for interfacial viscosity and PDI $(0.81)$, indicating a stronger linear relationship between interfacial viscosity and PDI $(P<0.05)$. In a previous study, the correlation between the rheological properties of the chitosan-PEO blends and their electrospinability can be seen (Rošic, et al., 2012). An interesting observation was that interfacial viscosity gave better response in predicting the electrospinability of the suspensions. All in all, the correlation between the rheological characteristics of the interface and nanofiber morphology is far more distinctive than the correlations with rheological properties in the bulk. 


\subsection{Properties of the produced particles}

Fig. 4 shows SEM images of the structures obtained together with the mean particle diameter and the polydispersity index (PDI) of the produced materials (Table 1). The mean diameter (Zav) of the produced particles was in the range of 120-390 nm for different compositions of BCNFS-WPI. In general, the diameter of the electrosprayed particles depended on the polymer concentration. An increase in both biopolymers causes an increase in the particle diameter and PDI, in accordance with previously published data (Wongsasulak, McClements, Yoovidhya \& Weiss, 2007). For example, the $z_{a v}$ of electrosprayed BCNFs-WPI particles increased from 121 to $148 \mathrm{~nm}$ by increasing the amount of BCNFsS from $1-16 \%$ wt and keeping the WPI constant (10\% wt).

Regarding the structure of the produced particles, the first observation is that particles with low WPI (10 -20\% wt) exhibit a more homogeneous structure. This could be explained by the binding of BCNFs monomers to the WPI backbone either through hydrophobic interactions or hydrogen bonding, forming a complex which affects intramolecular interactions and conformation of the protein. As previously hypothesized, interaction between BCNFs and protein may result in a more open molecular structure which may help to establish interactions with the WPI, decreasing the critical entanglement concentration and facilitating electrospraying (Kriegel, McClements \& Weiss, 2009).

The addition of higher WPI amount ( $30 \% \mathrm{wt})$ leads to the particles with multimodal size distributions and larger PDI values up to 3 (Table 1). Amorphous parts that are obvious in the micrographs of these materials are caused by the dripping of the BCNFs-WPI suspensions during the process. Albumins and globulins, the main components of WPI, have a globular structure which cause inter- and intra-molecular interactions (McClements, 2005). The mentioned $\alpha$-helical and $\beta$-sheet polymer chain configurations which may be adopted in higher WPI concentrations, also keep the rigidity of the chains, preventing the formation of monodispersed particles through electrospraying.

Principal component analysis (PCA) is conducted in order to evaluate the properties that mostly affect the size and structure of the particles (Fig. 5). In our case, 2 components have been extracted, since they have eigenvalues greater than or equal to 1.0. Together they account for $96.1 \%$ of the variability in our samples. As it can be seen, surface tension and 
1 conductivity are mostly affected by BCNFs, as their eigenvalues are parallel to the increase of

2 the BCNFs. This is in accordance with our previous measurements (3.1). On the other hand,

3 bulk and interfacial viscosity are weighted more heavily in a positive direction by WPI

4 concentration, as their eigenvalues are parallel to the augmentation of WPI. Another issue is

5 that the variables furthest from 0 , make the largest contribution to the system, which in our

6 case are interfacial viscosity and surface tension. Only with these properties the structure of

7 the electrosprayed particles could be successfully analyzed. Furthermore, a cluster analysis is

8 performed in order to categorize the samples (Fig. 5).

9

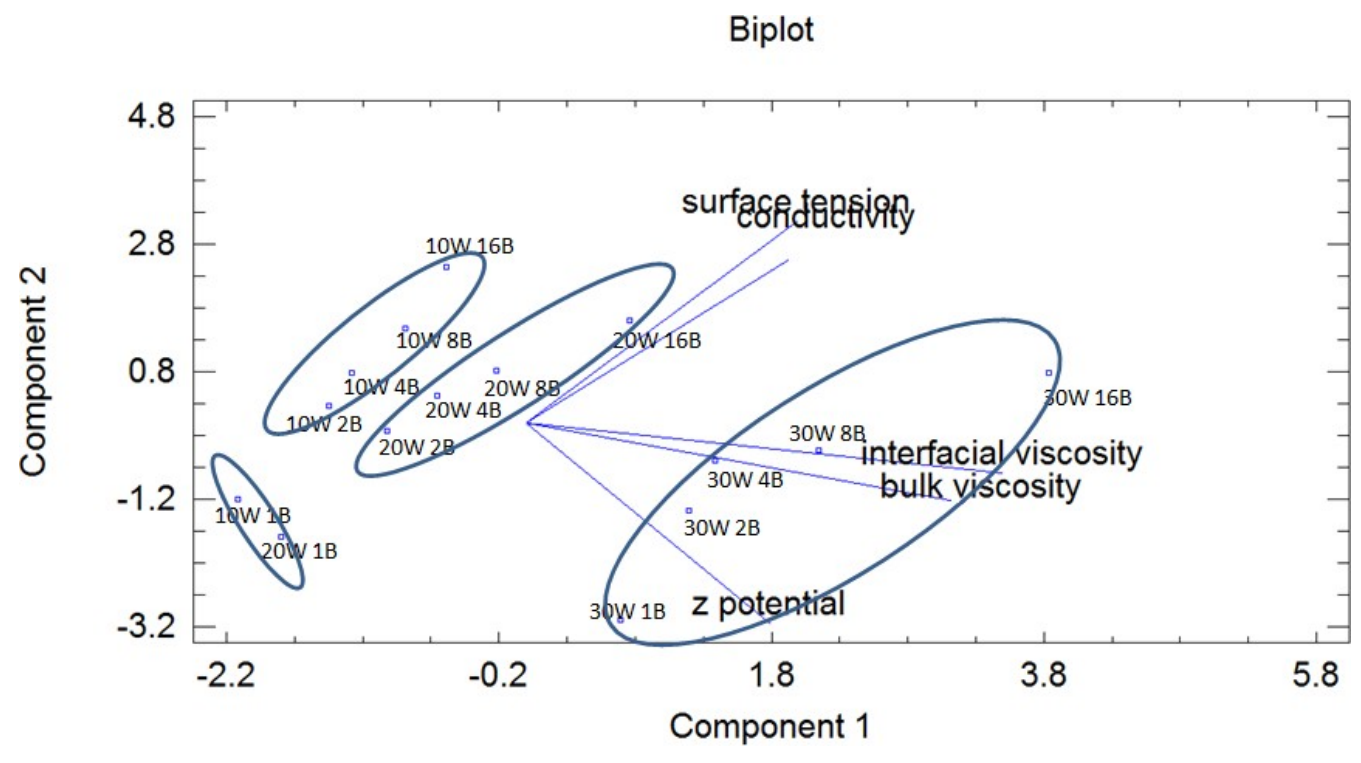

Fig. 5. Principal component analysis of the electrosprayed particles.

This procedure has created 4 clusters from the 15 observations supplied. Samples with $30 \%$ WPI comprise the first cluster, while the samples $10 \% \mathrm{WPI}-1 \% \mathrm{BCNFs}$ and $20 \% \mathrm{WPI}$ $1 \% \mathrm{BCNFs}$ the second. The samples with $10 \% \mathrm{WPI}$ and $2-16 \%$ BCNFs comprise the third cluster, and the samples with $20 \%$ WPI and $2-16 \%$ BCNFs the fourth cluster. This categorization indicates that WPI plays a key role on grouping the samples, especially in the first cluster, which leads to dripping suspensions. It is obvious that BCNFs in low concentrations also plays an important role in grouping (cluster 2). Cluster 3 also exhibits low 
1 viscosity and surface tension values, while cluster four (20\% WPI, 2-16\% BCNFs) yields

2 particles with the best properties.

3 In all cases, it was possible to produce particles with size distribution in the submicron range,

4 which is very promising as a first step of the encapsulation of bioactive compounds in the 5 BCNFS-WPI particles.

\section{Conclusions}

8 In this study, suspensions containing whey protein isolate (WPI) and bacterial cellulose (BC) in various concentrations (1-16\% wt BC and $10-30 \%$ wt WPI) were prepared and screened for their electrosprayability. Surface tension and electrical conductivity were found to increase as the BCNFs concentration increased, while they remained unaffected in terms of the protein concentration. The increase in surface tension and conductivity with increasing BCNFS concentration may be attributed to a change in the bonds that are formed between the polysaccharide and the proteins. Viscosity and interfacial viscosity increased as the proportion of BCNFS increased. A good correlation (0.83) was found between the interfacial rheology values of the suspension and the distribution of the produced particles. The produced particles varied in size (120 to $380 \mathrm{~nm}$ ) and polydispersity (PDI= 0.8-3). After a PCA analysis, it can be said that the properties that could best predict the structure of the electrosprayed particles, are interfacial viscosity and surface tension. The suspension that could be suggested as a good alternative material for microencapsulation is the one containing $8 \%$ BCNFS and $20 \% \mathrm{WPI}$. All in all, the results of this study are encouraging as a first step for the encapsulation of bioactive and volatile compounds in the BCNFs-WPI particles.

\section{Acknowledgements}

This work is part of the "Nonastru" project (11SYN-2-718), implemented within the National Strategic Reference Framework (NSRF) 2007-2013 and co- financed by National (Greek Ministry - General Secretariat of Research and Technology) and Community Funds (E.U.European Social Fund). Dr. C. Fasseas (Professor of Cell Biology, Faculty of Crop Science, 
Agricultural University of Athens) is acknowledged for SEM measurements. The authors would like to thank Arla Foods Hellas for kindly donating whey protein isolate.

\section{References}

Aceituno-Medina, M., Mendoza, S., Lagaron, J. M., López-Rubio, A. 2013. Development and characterization of food-grade electrospun fibers from amaranth protein and pullulan blends. Food Research International, 54(1), 667-674.

Azeredo, H.M.C. 2018 Bacterial cellulose for food applications-A review. International Journal in Advances in medical biotechnology, 1(2), 1-5

Bhardwaj, N., Kundu, S. C. 2010. Electrospinning: A fascinating fiber fabrication technique. Biotechnology Advances, 28(3), 325-347.

Bock, N., Dargaville, T. R., Woodruff, M. A. 2012. Electrospraying of polymers with therapeutic molecules: State of the art. Progress in Polymer Science, 37(11), 15101551.

Casper, C. L., Stephens, J. S., Tassi, N. G., Chase, D. B., Rabolt, J. F. 2004. Controlling Surface Morphology of Electrospun Polystyrene Fibers: Effect of Humidity and Molecular Weight in the Electrospinning Process. Macromolecules, 37(2), 573-578.

Choi S.M. \& Shin E.J. (2020) The Nanofication and Functionalization of Bacterial Cellulose and Its Applications. Nanomaterials, 10, 406; 1-24.

Freeman, J. 1996. Rheological Methods in Food Process Engineering. Freeman Press, 418.

Giroux, H. J., Robitaille, G., Britten, M. 2016. Controlled release of casein-derived peptides in the gastrointestinal environment by encapsulation in water-in-oil-in-water double emulsions. LWT - Food Science and Technology, 69, 225-232.

Gouzy, R., Tsekou, C., Remiln, C., Velikov, K.P. 2019. Cellulose microfibril networks in hydrolysed soy protein isolate solutions. Colloids and Surfaces A: Physicochemical and Engineering Aspects, 568, 277-283.

Hsieh, Y.-C., Yano, H., Nogi, M., Eichhorn, S.-J. 2008. An estimation of the Young's modulus of bacterial cellulose filaments. Cellulose, 15, 507-513.

Jia Y., Zhai X., Fu W., Liu Y., Li F., Zhong C., 2016, Surfactant-free emulsions stabilized by tempo-oxidized bacterial celluloseCarbohydrate Polymers, 151 (20), 907-915.

Kaltsa, O., Paximada, P., Mandala, I., Scholten, E. 2014. Physical characteristics of submicron emulsions upon partial displacement of whey protein by a small molecular weight surfactant and pectin addition. Food Research International, 66(0), 401-408.

Koupantsis, T., Pavlidou, E., Paraskevopoulou, A. 2016. Glycerol and tannic acid as applied in the preparation of milk proteins - CMC complex coavervates for flavour encapsulation. Food Hydrocolloids, 57, 62-71.

Kriegel, C., Kit, K. M., McClements, D. J., Weiss, J. 2009. Electrospinning of chitosanpoly(ethylene oxide) blend nanofibers in the presence of micellar surfactant solutions. Polymer, 50(1), 189-200.

Li, Q., Wang, Y., Wu, Y., He, K., Li, Y., Luo, X., Li, B., Wang, C., Liu, S. 2019. Flexible cellulose nanofibrils as novel Pickering stabilizers: The emulsifying property and packing behavior. Food Hydrocolloids, 88, 180-189.

McClements, D. J. 2005. Food Emulsions: Principles, Practice and Techniques. Boca Raton, CRC Press.

Neo, Y. P., Ray, S., Jin, J., Gizdavic-Nikolaidis, M., Nieuwoudt, M. K., Liu, D., Quek, S. Y. 2013. Encapsulation of food grade antioxidant in natural biopolymer by electrospinning technique: a physicochemical study based on zein-gallic acid system. Food Chem, 136(2), 1013-1021. 
Nishi, Y., Uryu, M., Yamanaka, S., Watanabe, K., Kitamura, N., Iguchi, M. 1990. The structure and mechanical properties of sheets prepared from bacterial cellulose. Journal of Materials Science, 25 (6), 2997-3001.

Panagopoulou, E., Tsouko, E., Kopsahelis, N., Koutinas, A., Mandala, I., Evageliou, V. 2015. Olive oil emulsions formed by catastrophic phase inversion using bacterial cellulose and whey protein isolate. Colloids and Surfaces A: Physicochemical and Engineering Aspects, 486, 203-210.

Pancholi, K., Ahras, N., Stride, E., Edirisinghe, M. 2009. Novel electrohydrodynamic preparation of porous chitosan particles for drug delivery. J Mater Sci Mater Med, 20(4), 917-923.

Paximada, P., Dimitrakopoulou, E. A., Tsouko, E., Koutinas, A. A., Fasseas, C., Mandala, I. G. 2016a. Structural modification of Bacterial Cellulose fibrils under ultrasonic irradiation. Carbohydrate Polymers.

Paximada, P., Koutinas, A. A., Scholten, E., Mandala, I. G. 2016b. Effect of bacterial cellulose addition on physical properties of WPI emulsions. Comparison with common thickeners. Food Hydrocolloids, 54, Part B, 245-254.

Paximada, P., Tsouko, E., Kopsahelis, N., Koutinas, A. A., Mandala, I. 2016c. Bacterial cellulose as stabilizer of o/w emulsions. Food Hydrocolloids, 53, 225-232.

Paximada, P., Dubey, B., Howarth, M. 2018. Electrosprayed particles from nano-emulsions as carriers of fish oil. Techconnect Briefs, 1-4.

Pelipenko, J., Kristl, J., Rosic, R., Baumgartner, S., Kocbek, P. 2012. Interfacial rheology: an overview of measuring techniques and its role in dispersions and electrospinning. Acta Pharm, 62(2), 123-140.

Regev, O., Vandebril, S., Zussman, E., Clasen, C. 2010. The role of interfacial viscoelasticity in the stabilization of an electrospun jet. Polymer, 51(12), 2611-2620.

Renou, F., Petibon, O., Malhiac, C., Grisel, M. 2013. Effect of xanthan structure on its interaction with locust bean gum: Toward prediction of rheological properties. Food Hydrocolloids, 32(2), 331-340.

Rošic, R., Pelipenko, J., Kocbek, P., Baumgartner, S., Bešter-Rogač, M., Kristl, J. 2012. The role of rheology of polymer solutions in predicting nanofiber formation by electrospinning. European Polymer Journal, 48(8), 1374-1384.

Sarhan, W. A., \& Azzazy, H. M. E. 2015. High concentration honey chitosan electrospun nanofibers: Biocompatibility and antibacterial effects. Carbohydrate Polymers, 122, 135-143.

Theron, S. A., Zussman, E., Yarin, A. L. 2004. Experimental investigation of the governing parameters in the electrospinning of polymer solutions. Polymer, 45(6), 2017-2030.

Winuprasith, T., Suphantharika, M. (2013). Microfibrillated cellulose from mangosteen (Garcinia mangostana L.) rind: Preparation, characterization, and evaluation as an emulsion stabilizer. Food Hydrocolloids, 32(2), 383-394.

Wongsasulak, S., Kit, K. M., McClements, D. J., Yoovidhya, T., Weiss, J. 2007. The effect of solution properties on the morphology of ultrafine electrospun egg albumen-PEO composite fibers. Polymer, 48(2), 448-457.

Yu, D.-G., Branford-White, C., Williams, G. R., Bligh, S. W. A., White, K., Zhu, L.-M., Chatterton, N. P. 2011. Self-assembled liposomes from amphiphilic electrospun nanofibers. Soft Matter, 7(18), 8239-8247.

Zhang, X., Liu, Y., Wang, Y., Luo, X., Li, Y. Li, B. 2019. Surface modification of cellulose nanofibrils with protein nanoparticles for enhancing the stabilization of $\mathrm{O} / \mathrm{W}$ pickering emulsions. Food Hydrocolloids, 97, 105-180. 
Dr. P. Paximada was responsible for conceptualization, data curation, investigation, and writing the original draft, Mrs E. Kanavou was responsible for data curation and Dr. I. Mandala was responsible for

conceptualization, supervision, writing-review \& editing

${ }^{\star}$ Credit Author Statement 